\title{
Association between increased signal intensity at the proximal patellar tendon and patellofemoral geometry in community-based asymptomatic middle- aged adults: a cross-sectional study
}

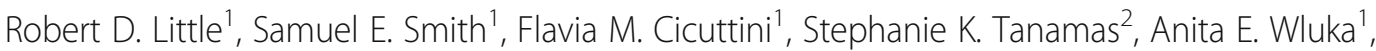
Sultana Monira Hussain ${ }^{1}$, Donna M. Urquhart ${ }^{1}$, Graeme Jones ${ }^{3}$ and Yuanyuan Wang ${ }^{1 *}$

\begin{abstract}
Background: Histological and epidemiological data suggest that increased signal intensity at the proximal patellar tendon on magnetic resonance imaging is a response to tendon loading. As patellofemoral geometry is a mediator of loading, we examined the association between patellofemoral geometry and the prevalence of increased signal intensity at the patellar tendon in community-based middle-aged adults.

Methods: Two hundred-one adults aged 25-60 years in a study of obesity and musculoskeletal health had the patellar tendon assessed from magnetic resonance imaging. Increased signal intensity at the proximal patellar tendon was defined as hyper-intense regions of characteristic pattern, size and distribution on both T1- and T2weighted sequences. Indices of patellofemoral geometry, including Insall-Salvati ratio, patellofemoral congruence angle, sulcus angle, and lateral condyle-patella angle, were measured from magnetic resonance imaging using validated methods. Binary logistic regression was used to examine the association between patellofemoral geometrical indices and the prevalence of increased signal intensity at the patellar tendon.
\end{abstract}

Results: The prevalence of increased signal intensity at the patellar tendon was 37.3\%. A greater Insall-Salvati ratio (odds ratio $0.80,95 \%$ confidence interval $0.66-0.97$ per 0.1 change in the ratio, $p=0.02$ ), indicative of a higher-riding patella, and a larger patellofemoral congruence angle (odds ratio 0.91, 95\% confidence interval 0.85-0.98 per 5 degree change in the angle, $p=0.01$ ), indicating a more laterally placed patella, were associated with reduced odds of increased signal intensity at the patellar tendon. Sulcus angle and lateral condyle-patella angle were not significantly associated with the odds of increased signal intensity at the patellar tendon.

(Continued on next page)

* Correspondence: yuanyuan.wang@monash.edu

${ }^{1}$ School of Public Health and Preventive Medicine, Monash University, 553 St Kilda Road, Melbourne, VIC 3004, Australia

Full list of author information is available at the end of the article

C C The Author(s). 2020 Open Access This article is licensed under a Creative Commons Attribution 4.0 International License, which permits use, sharing, adaptation, distribution and reproduction in any medium or format, as long as you give appropriate credit to the original author(s) and the source, provide a link to the Creative Commons licence, and indicate if changes were made. The images or other third party material in this article are included in the article's Creative Commons licence, unless indicated otherwise in a credit line to the material. If material is not included in the article's Creative Commons licence and your intended use is not permitted by statutory regulation or exceeds the permitted use, you will need to obtain permission directly from the copyright holder. To view a copy of this licence, visit http://creativecommons.org/licenses/by/4.0/. The Creative Commons Public Domain Dedication waiver (http://creativecommons.org/publicdomain/zero/1.0/) applies to the data made available in this article, unless otherwise stated in a credit line to the data. 
(Continued from previous page)

Conclusions: In community-based asymptomatic middle-aged adults, increased signal intensity at the patellar tendon was common and associated with Insall-Salvati ratio and patellofemoral congruence angle, suggesting a biomechanical mechanism. Such work is likely to inform tissue engineering and cell regeneration approaches to improving outcomes in those with tendon pathology.

Keywords: Patellar tendon, Insall-Salvati ratio, Patellofemoral congruence angle, Magnetic resonance imaging

\section{Background}

The structure-function relationships in articular cartilage and subchondral bone have been widely investigated, but relatively little attention has been given to tendons. The increasing interest and effort in cell regeneration therapy and tissue engineering to promote tendon repair and create artificial tendons $[1,2]$ highlight the need for such work to be underpinned by a comprehensive understanding of the functional morphology of tendons and the risk factors for tendon abnormalities.

Magnetic resonance imaging (MRI) enables noninvasive assessment of joint structures with signal alterations reflecting tissue composition. Histological examination of increased signal intensity of the proximal patellar tendon on T1-weighted and fluid-sensitive MRI have been shown to be due to invaginated adipose tissue, vessels, and perivascular connective tissue [3]. We have shown increased signal intensity at the proximal patellar tendon to be common in otherwise asymptomatic community-based adults aged over 40 years and to be associated with obesity, high levels of physical activity and increased size of vastus medialis, suggesting a predominant biomechanical mechanism $[4,5]$. These findings, together with the histological changes within the region of increased signal intensity at the proximal patellar tendon, support a tissue response to increased loading in the patellar tendon.

Patellofemoral geometry has an important role on the direction and magnitude of forces acting on the patellofemoral joint and patellofemoral alignment may affect load distribution to the extensor mechanism, thus altering the distribution of strain within the patellar tendon upon quadriceps contraction [6, 7]. Given the potential biomechanical mechanism for the presence of increased signal intensity at the patellar tendon $[4,5]$, we examined the association between patellofemoral geometry and the prevalence of increased signal intensity at the proximal patellar tendon in community-based asymptomatic middle-aged individuals. We hypothesised that altered patellofemoral geometry would be associated with an increased odds of increased signal intensity at the proximal patellar tendon.

\section{Methods}

\section{Study participants}

Two hundred and fifty participants, aged 25-60 years and across the spectrum from normal weight to obese, were recruited into a study investigating the effect of obesity and weight loss on knee health. They were recruited via advertising in the local media, hospital waiting rooms, community weight loss organisations, and private weight loss and obesity clinics [8]. Participants were excluded if they had a history of any joint disease diagnosed by a medical practitioner, significant knee injury requiring non-weight-bearing therapy, previous knee surgery including arthroscopy, knee pain requiring prescribed analgesia or no weight-bearing activity for > $24 \mathrm{~h}$, contraindication to MRI, or malignancy. The Monash University Human Research Ethics Committee and the Alfred Hospital Ethics Committee approved this study. Written informed consent was obtained from all the participants.

\section{Assessment of anthropometry and physical activity}

Weight was measured using a single pair of electronic scales to the nearest $0.1 \mathrm{~kg}$ (shoes and bulky clothing removed). Height was measured using a stadiometer to the nearest $0.1 \mathrm{~cm}$ (shoes removed). Body mass index (BMI) was calculated $\left(\mathrm{kg} / \mathrm{m}^{2}\right)$. Strenuous physical activity was assessed as previously described [9] by asking "On how many days during the last 14 days did you spend at least 20 minutes doing strenuous exercise that was severe enough to raise your pulse rate, cause you to breathe faster (e.g. bicycling, brisk walking, jogging and aerobics)". The participants were asked to select one from the five frequency options: no days; $1-2$ days; $3-5$ days; $6-8$ days; and $\geq 9$ days. Individuals were classified as performing strenuous physical activity if they participated in strenuous activity for $\geq 3$ days during the previous 14 days.

\section{Acquisition of MRI}

Participants underwent an MRI of their dominant knee (the lower limb from which the individual steps off from when starting to walk) on a 1.5 - $\mathrm{T}$ whole body magnetic resonance unit (Philips, Medical Systems, Eindhoven, the Netherlands) using a commercial transmit-receive extremity coil, with two sequences obtained: (1) T1weighted, fat-saturated 3D gradient recall acquisition in the steady state, repetition time $58 \mathrm{~ms}$, echo time $12 \mathrm{~ms}$, flip angle 55 degrees, field of view $16 \mathrm{~cm}, 60$ partitions, $512 \times 512$ matrix, one acquisition. Sagittal images had a 
partition thickness of $1.5 \mathrm{~mm}$ and an in-plane resolution of $0.31 \times 0.31 \mathrm{~mm}$. (2) Coronal T2-weighted, fatsaturated, fast spin echo acquisition, repetition time $2200 \mathrm{~ms}$, echo time $20 / 80 \mathrm{~ms}$, flip angle 90 degrees, slice thickness $3 \mathrm{~mm}$ with a 0.3 inter-slice gap, 1 excitation, field of view $13 \mathrm{~cm}$, and $256 \times 192$ matrix. MRI measurements were undertaken by independent observers who had at least two years' experience in MRI measurement and were trained by a radiologist and blinded to participant characteristics.

\section{Assessment of increased signal intensity at the proximal patellar tendon}

Increased signal intensity was assessed as present or absence at the proximal patellar tendon. The presence of increased signal intensity at the proximal patellar tendon was defined as a hyper-intense area with characteristic distribution, size, and pattern present on at least two contiguous slices in the proximal region of the inferior patellar tendon. This was assessed on sagittal T1weighted fat-saturated images and then confirmed on coronal T2-weighted fat-saturated images in order to reduce the false positives due to the 'magic angle effect' [10] (Fig. 1). The reproducibility of the measurement was assessed using 50 randomly selected MRIs, and the intra- and inter-observer reproducibility (Cohen's kappa) was 0.90 and 0.82 , respectively.

\section{Measurement of patellofemoral geometry}

Insall-Salvati ratio, sulcus angle, patellofemoral congruence angle, and lateral condyle-patella angle, were measured by image processing using the Osiris software
(University of Geneva, Switzerland). Insall-Salvati ratio measures patellar height, which represents the ratio of the length of the patellar tendon to the length of the patella, measured from the sagittal plane at the midpoint of the patella (Fig. 2 a). A ratio $>1.2$ corresponds to a high-riding patella and a ratio $<0.8$ indicates a low-riding patella [11]. Our intra-observer reproducibility [intra-class correlation coefficient (ICC)] was 0.86 [8]. Sagittal MR images were reformatted to axial images with a resolution of $0.31 \times$ $0.31 \mathrm{~mm}$. The femoral sulcus angle was formed by lines connecting the lowest point of the intercondylar sulcus and the highest points of the bony medial and lateral femoral condyles (Fig. 2 b). Larger angles correspond to a shallower articular surface and smaller angles correspond to a deeper articular surface. Our intra-observer reproducibility (ICC) was 0.98 [8]. The patellofemoral congruence angle is an index of patellar subluxation defining the relationship between the patellar articular ridge and the intercondylar sulcus [12]. The sulcus angle was bisected by a neutral reference line, and the measured line was projected from the apex of the sulcus angle to the lowest articular ridge of the patella. A positive value was assigned if the measured line fell lateral to the reference line (Fig. 2 c), with angles above 16 degrees of lateral subluxation corresponding to abnormal lateral patella position. Our intraobserver reproducibility (ICC) was 0.99 . The lateral condyle-patella angle was measured as the angle between the bony posterior femoral condyles and the bony lateral patella facet (Fig. $2 \mathrm{~d}$ ), with larger angles indicating more medial patellar inclination and smaller angles indicating more lateral patellar inclination. Our intra-observer reproducibility (ICC) was 0.98 [8]. The three angles described

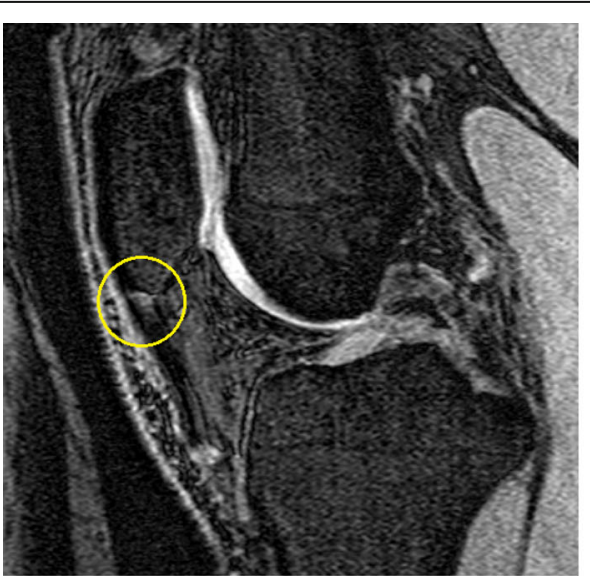

a. Increased signal intensity at the proximal patellar tendon on T1-weighted fat-saturated sagittal MRI

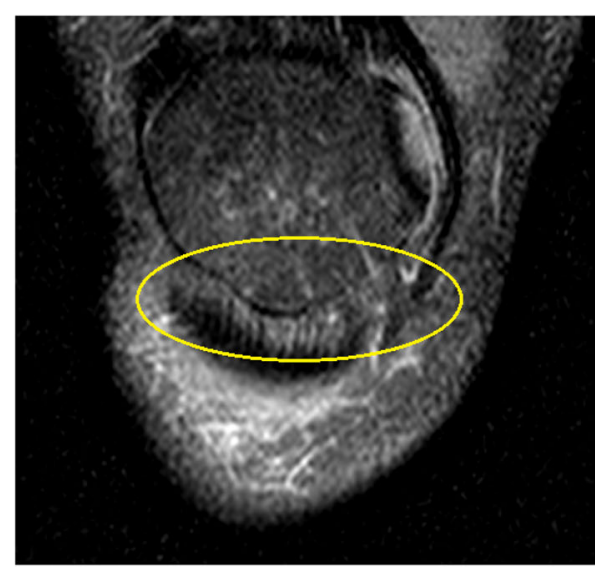

b. Increased signal intensity at the proximal patellar tendon on T2-weighted fat-saturated coronal MRI

Fig. 1 Increased signal intensity at the proximal patellar tendon on a. T1-weighted fat-saturated sagittal MRI; and b. T2-weighted fat-saturated coronal MRI 


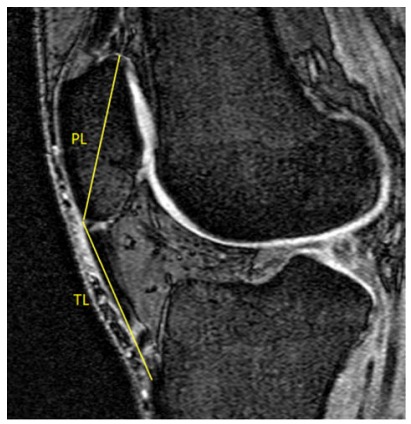

a Insall-Salvati ratio was calculated as a ratio of the patellar tendon length (TL) : the patella length (PL)

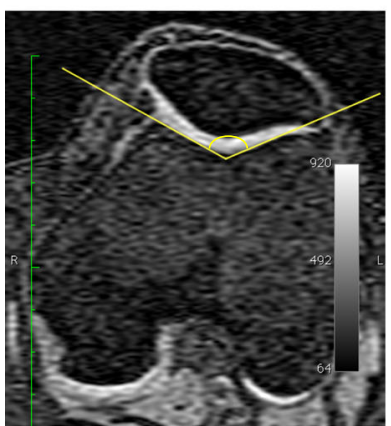

b Sulcus angle was defined as the angle formed between lines joining the highest points of the bony medial and lateral condyles and the lowest bony point of the intercondyla sulcus

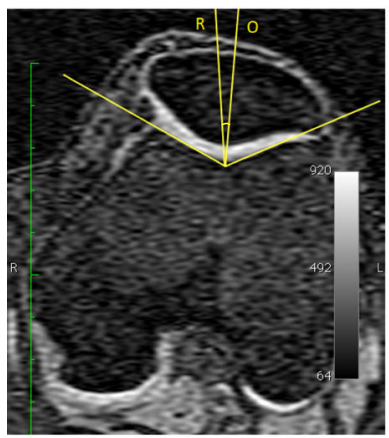

c Patellofemoral congruence angle. The sulcus angle was bisected by a neutral reference line $(\mathrm{O})$, and the measured line was projected from the apex of the sulcus angle to the lowest articular ridge of the patella $(\mathrm{R})$

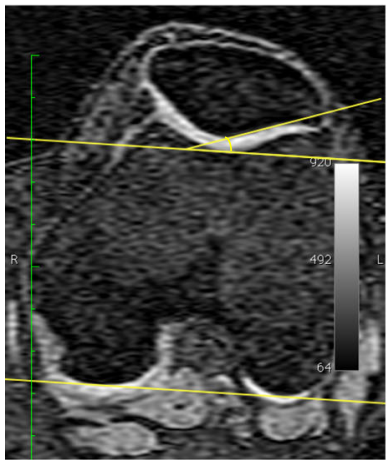

d Lateral condyle-patella angle was measured as the angle between the bony posterior femoral condyles and the bony lateral patella facet

Fig. 2 Measurement of patellofemoral geometry above were all measured in degrees at the mid-patella level.

\section{Statistical analysis}

The characteristics of participants with and without increased signal intensity at the patellar tendon were compared using independent samples t-test or chi square test when appropriate. Binary logistic regression was used to examine the relationship between patellofemoral geometrical indices (independent variable) and the prevalence of increased signal intensity at the patellar tendon (dependent variable), with multivariable analyses adjusted for age, gender and BMI. Patellofemoral geometry variables were entered in the regression models as continuous variables with some conversions: InsallSalvati ratio was entered as per 0.1 (i.e raw data multiplied by 10), patellofemoral congruence angle, sulcus angle, and lateral condyle-patella angle were entered as per 5 degrees (i.e. raw data divided by 5). A $p$-value of less than 0.05 (two-tailed) was considered statistically significant. All analyses were performed using the IBM SPSS statistical package (standard version 24, SPSS, Chicago, IL, USA).

\section{Results}

Among the 250 participants, increased signal intensity at the patellar tendon could be assessed in 201 (80.4\%) participants, with 49 participants without the assessment due to the unavailability of particular T2-weighted coronal images to confirm the presence of increased signal intensity at the patellar tendon. These 49 participants had higher BMI [37.8 (SD 9.7) vs. 33.2 (SD 9.3) kg/m², $p=0.003$ ] and greater Insall-Salvati ratio [1.06 (SD 0.14) vs. 0.98 (SD 0.16), $p=0.01$ ] compared with those with patellar tendon assessment.

Participants with increased signal intensity at the patellar tendon $(n=75,37.3 \%)$ were more likely to be male (33\% vs. $20 \%, p=0.03$ ), had lower Insall-Salvati ratio (0.94 vs. $1.00, p=0.02)$ and smaller patellofemoral congruence angle (10.3 vs. 17.6 degrees, $p=0.04$ ), compared with those without increased signal intensity at the patellar tendon (Table 1). No significant differences were found for age, BMI, sulcus angle, or lateral condylepatella angle between the two groups.

In univariable analyses, a higher Insall-Salvati ratio and a greater patellofemoral congruence angle were associated with reduced odds of increased signal intensity at the patellar tendon (Table 2). After adjustment for age, gender and BMI, a greater Insall-Salvati ratio [odds ratio $0.80,95 \%$ confidence interval $0.66-0.97, p=0.02$ ] and a larger patellofemoral congruence angle (odds ratio $0.91,95 \%$ confidence interval $0.85-0.98, p=0.01$ ) remained significantly associated with decreased odds of increased signal intensity at the patellar tendon. There 
Table 1 Characteristics of study participants

\begin{tabular}{|c|c|c|c|}
\hline & $\begin{array}{l}\text { No increased signal intensity } \\
\text { at patellar tendon }(n=126)\end{array}$ & $\begin{array}{l}\text { Increased signal intensity } \\
\text { at patellar tendon }(n=75)\end{array}$ & $P$ \\
\hline Age, years & $46.3(9.3)$ & $46.3(9.7)$ & 0.98 \\
\hline Female, n (\%) & $101(80.2)$ & $50(66.7)$ & 0.03 \\
\hline Body mass index, $\mathrm{kg} / \mathrm{m}^{2}$ & $33.6(9.5)$ & $32.6(9.1)$ & 0.49 \\
\hline Strenuous physical activity, n (\%) & $100(79.4)$ & $59(78.7)$ & 0.91 \\
\hline \multicolumn{4}{|l|}{ Patellofemoral geometrical indices } \\
\hline Insall-Salvati ratio & $1.00(0.16)$ & $0.94(0.16)$ & 0.02 \\
\hline Patellofemoral congruence angle, degrees & $17.6(24.4)$ & $10.3(23.2)$ & 0.04 \\
\hline Sulcus angle, degrees & $150.9(10.1)$ & $149.9(9.5)$ & 0.48 \\
\hline Lateral condyle-patella angle, degrees & $19.4(7.2)$ & $19.1(6.6)$ & 0.81 \\
\hline
\end{tabular}

Data presented as mean (SD) or no (\%)

was no significant association for sulcus angle or lateral condyle-patella angle in either univariable or multivariable analysis (Table 2). Adding strenuous physical activity in the regression models did not change the results (data not shown).

\section{Discussion}

This study showed that increased signal intensity at the proximal patellar tendon on T1-weight and fluidsensitive MRI was present in over one third of community-based, asymptomatic middle-aged individuals. A greater Insall-Salvati ratio, indicating a highriding patella, and a larger patellofemoral congruence angle, reflecting a more laterally placed patella (subluxation), were associated with reduced odds of increased signal intensity at the proximal patellar tendon.

The high prevalence of increased signal intensity at the proximal patellar tendon in our study provides an opportunity to understand patellar tendon health given that this MRI change is known to represent distinct pathological processes of invaginated adipose tissue, vessels, and perivascular connective tissue [3]. A highriding patella and a laterally placed patella have been associated with a range of patellofemoral disorders including radiographic patellofemoral osteoarthritis [13], MRI-derived structural features of patellofemoral osteoarthritis (such as bone marrow lesions and cartilage damages) and their worsening [14-16], and recurrent patellar dislocation [17]. Our study found reduced odds of increased signal intensity at the proximal patellar tendon in relation to a greater Insall-Salvati ratio in middle-aged, asymptomatic, predominantly obese adults. Redistribution of mechanical load would be one potential mechanism to explain our result, as it has been suggested that a high-riding patella may increase anteroposterior load bearing through the patellar cartilage, which in turn offloads patellar tendon stress, leading to reduced connective tissue damage and less signal abnormality within the patellar tendon.

The reduced odds of increased signal intensity at the patellar tendon related to a larger patellofemoral congruence angle observed in our study is supported by our previous study reporting an association between greater vastus medialis cross-sectional area and higher prevalence of increased signal intensity at the patellar tendon [5]. Greater vastus medialis activity reduces lateral patella stress via increased medial tension [18], and greater vastus medialis size may therefore reduce lateral patella maltracking and patellofemoral congruence angle which diminishes articular cartilage stress and thereby ameliorates any reduction in patellar tendon load. The relationship observed in our study between increased signal intensity at the proximal patellar tendon and InsallSalvati ratio and patellofemoral congruence angle suggests a biomechanical mechanism for its pathogenesis. Altering patellofemoral joint mechanics by targeting

Table 2 Association between patellofemoral geometrical indices and the prevalence of increased signal intensity at the patellar tendon

\begin{tabular}{|c|c|c|c|c|}
\hline & $\begin{array}{l}\text { Univariable analysis } \\
\text { Odds ratio ( } 95 \% \mathrm{Cl})\end{array}$ & $P$ value & $\begin{array}{l}\text { Multivariable analysis* } \\
\text { Odds ratio }(95 \% \mathrm{Cl})\end{array}$ & $P$ value \\
\hline Insall-Salvati ratio (per 0.1) & $0.80(0.66,0.97)$ & 0.02 & $0.80(0.66,0.97)$ & 0.02 \\
\hline Patellofemoral congruence angle (per 5 degrees) & $0.94(0.88,1.00)$ & 0.04 & $0.91(0.85,0.98)$ & 0.01 \\
\hline Sulcus angle (per 5 degrees) & $0.95(0.82,1.10)$ & 0.48 & $0.95(0.82,1.11)$ & 0.52 \\
\hline Lateral condyle-patella angle (per 5 degrees) & $0.98(0.79,1.20)$ & 0.81 & $0.98(0.79,1.21)$ & 0.82 \\
\hline
\end{tabular}


patellofemoral geometry may provide a potential approach to improving patellar tendon morphology and treatment outcomes for people with tendon pathology.

Limitations of our study include the cross-sectional analyses for the association between patellofemoral geometry and increased signal intensity at the proximal patellar tendon, and thus the causality cannot be examined. While highly reproducible, the increased signal intensity at the proximal patellar tendon detected from MRI may not correlate with clinical disease [19, 20]. However, longitudinal studies have demonstrated that abnormalities within asymptomatic tendon identified by ultrasound predicted the subsequent development of tendon-related symptoms and disability [21, 22]. Therefore, the increased signal intensity at the patellar tendon in asymptomatic participants may be demonstrating the early phase of structural changes in the patellar tendon in reaction to biomechanical factors. In this study femoral sulcus angle, patellofemoral congruence angle, and lateral condyle-patella angle were measured on axial images. Although there was some loss of information when reformatting the original sagittal images to the axial images, the axial images had a good resolution of $0.31 \times$ $0.31 \mathrm{~mm}$. All the angle measurements were based on the anatomical landmarks of bone and cartilage which were clearly shown on the axial images. Furthermore, all the images were processed in the same way and all the angle measurements were performed in the same way for all the participants with high reproducibility. Therefore the reformatting of images is unlikely to have significantly affected the angle measurements or the results of the study. Participants in our study underwent non-weightbearing geometrical measurements of the patellofemoral joint with the knee in full extension which is a suboptimal representation of joint motion and weight-bearing in day-to-day activity [23]. Traditionally, patellofemoral geometry was first measured on plain radiographs. Insall-Salvati ratio was measured on lateral radiographs [11], and femoral sulcus angle [24], patellofemoral congruence angle [12], and lateral condyle-patella angle [25] were measured on skyline view radiographs, all with patients in a non-weight-bearing position. Although the measurement of patellofemoral alignment would be expected to be affected by the patient position (supine, prone, weight-bearing), the evidence is inconclusive regarding the potential difference in patellofemoral alignment between the weight-bearing and non-weightbearing conditions [26]. In this study all participants were assessed non-weight-bearing. While the absolute values of patellofemoral geometry measures might be different based on weight-bearing or not, this is unlikely to have affected our findings of the association of greater Insall-Salvati ratio and patellofemoral congruence angle with reduced odds of increased signal intensity at the patellar tendon as all participants were measured in the same way and any measurement error is likely to be non-differential which would result in an underestimation of the magnitude of observed associations. InsallSalvati ratio is susceptible to measurement errors arising from variations in patellar position and morphology [27, 28]. The strengths of our study include the enrollment of community-based participants across a wide range of BMI, thereby increasing the generalizability of our findings. In addition, we used a non-invasive, MRI-based assessment of the patellar tendon using both T1weighted and fluid-sensitive sequences to reduce the risk of erroneously misclassifying 'magic angle' signal intensity as true structural alterations in the patellar tendon.

\section{Conclusions}

Our study showed a high prevalence of MRI-detected increased signal intensity at the proximal patellar tendon in a sample of community-based middle-aged asymptomatic adults across a range of BMI. The lower odds of increased signal intensity at the patellar tendon in relation to greater Insall-Salvati ratio and patellofemoral congruence angle supports biomechanical mechanisms. Such work is likely to inform tissue engineering and cell regeneration approaches to improving patellar tendon morphology and outcomes in those with tendon pathology by targeting patellofemoral geometry to alter patellofemoral joint mechanisms.

\section{Abbreviations \\ BMI: Body mass index; Cl: Confidence interval; ICC: Intraclass correlation coefficient; MRI: Magnetic resonance imaging}

\section{Acknowledgements}

We would like to thank Judy Hankin for recruitment of study participants, the MRI unit at the Epworth Hospital for their cooperation, and Kevin Morris for technical support. A special thank you to all the study participants who made this study possible.

\section{Authors' contributions}

RDL was involved in data acquisition, performed data analysis, and drafted the manuscript. FMC was involved in conception and design of the study. SES, SKT, AEW, and GJ were involved in data acquisition. YW was involved in conception and design of the study, data analysis, and drafted the manuscript. RDL, SES, FMC, SKT, AEW, SMH, DMU, GJ, and YW contributed to interpretation of data, reviewed the manuscript critically for important intellectual content, and approved the final manuscript. YW had full access to all the data and take responsibility for the integrity of the data and the accuracy of the data analysis. The author(s) read and approved the final manuscript.

\section{Funding}

This study was funded by a grant from the National Health and Medical Research Council (NHMRC). AEW and YW are the recipients of NHMRC Translating Research into Practice Fellowship (APP1150102 and APP1168185, respectively). SMH is the recipient of NHMRC Early Career Fellowship (APP1142198). DMU is the recipient of NHMRC Career Development Fellowship (Level 2, APP1142809). The NHMRC was not involved in the design of the study and collection, analysis, and interpretation of data and in writing the manuscript. 


\section{Availability of data and materials}

All data generated or analysed during this study are included in this published article [and its supplementary information files].

\section{Ethics approval and consent to participate}

Ethical approval was granted by the Alfred Hospital Ethics Committee and Monash University Human Research Ethics Committee. All participants provided written informed consent prior to commencement of the study.

\section{Consent for publication}

Not applicable.

\section{Competing interests}

The authors declare that they have no competing interests. Anita Wluka is a Section Editor for the journal; Flavia Cicuttini and Yuanyuan Wang are Associate Editors for the journal.

\section{Author details}

'School of Public Health and Preventive Medicine, Monash University, 553 St Kilda Road, Melbourne, VIC 3004, Australia. ${ }^{2}$ Institute of Vector-Borne Disease, Monash University, Clayton, VIC 3800, Australia. ${ }^{3}$ Menzies Institute for Medical Research, University of Tasmania, Hobart, TAS 7000, Australia.

Received: 22 January 2020 Accepted: 13 August 2020

Published online: 22 August 2020

\section{References}

1. Zhang AY, Chang J. Tissue engineering of flexor tendons. Clin Plast Surg. 2003;30:565-72.

2. Chamberlain CS, Saether EE, Aktas E, Vanderby R. Mesenchymal stem cell therapy on tendon/ligament healing. J Cytokine Biol. 2017;2:112.

3. Yun SJ, Jin W, Park YK, Kim GY, Yoon SH, Park SY, Lee JE, Park JS, Ryu KN. Increased signal intensity at the proximal patellar tendon: correlation between MR imaging and histology in eight cadavers and clinical MR imaging studies. Eur Radiol. 2015;25:2976-83.

4. Fairley J, Toppi J, Cicuttini FM, Wluka AE, Giles GG, Cook J, O'Sullivan R, Wang $Y$. Association between obesity and magnetic resonance imaging defined patellar tendinopathy in community-based adults: a cross-sectional study. BMC Musculoskelet Disord. 2014;15:266.

5. Toppi J, Fairley J, Cicuttini FM, Cook J, Davis SR, Bell RJ, Hanna F, Wang Y. Factors associated with magnetic resonance imaging defined patellar tendinopathy in community-based middle-aged women: a prospective cohort study. BMC Musculoskelet Disord. 2015;16:184

6. Makhsous M, Lin F, Koh JL, Nuber GW, Zhang LQ. In vivo and noninvasive load sharing among the vasti in patellar malalignment. Med Sci Sports Exerc. 2004;36:1768-75.

7. Mohr KJ, Kvitne RS, Pink MM, Fideler B, Perry J. Electromyography of the quadriceps in patellofemoral pain with patellar subluxation. Clin Orthop Relat Res. 2003:261-71.

8. Tanamas SK, Teichtahl AJ, Wluka AE, Wang Y, Davies-Tuck M, Urquhart DM, Jones G, Cicuttini FM. The associations between indices of patellofemoral geometry and knee pain and patella cartilage volume: a cross-sectional study. BMC Musculoskelet Disord. 2010;11:87.

9. Berry PA, Wluka AE, Davies-Tuck ML, Wang Y, Strauss BJ, Dixon JB, Proietto J, Jones $\mathrm{G}$, Cicuttini FM. Sex differences in the relationship between bone mineral density and tibial cartilage volume. Rheumatology (Oxford). 2011;50:563-8.

10. Karantanas AH, Zibis AH, Papanikolaou N. Increased signal intensity on fatsuppressed three-dimensional T1-weighted pulse sequences in patellar tendon: magic angle effect? Skelet Radiol. 2001;30:67-71.

11. Insall J, Salvati E. Patella position in the normal knee joint. Radiology. 1971; 101:101-4.

12. Merchant AC, Mercer RL, Jacobsen RH, Cool CR. Roentgenographic analysis of patellofemoral congruence. J Bone Joint Surg Am. 1974;56:1391-6.

13. Kalichman L, Zhang Y, Niu J, Goggins J, Gale D, Felson D, Hunter D. The association between patellar alignment on magnetic resonance imaging and radiographic manifestations of knee osteoarthritis. Arthritis Res Ther. 2007;9:R26.

14. Haj-Mirzaian A, Guermazi A, Pishgar F, Pourvaziri A, Roemer FW, Sereni C, Hakky M, Zikria B, Stefanik JJ, Demehri S. Association of patella Alta with worsening of patellofemoral osteoarthritis-related structural damage: data from the osteoarthritis initiative. Osteoarthr Cartil. 2019;27:278-85.

15. Stefanik JJ, Zhu Y, Zumwalt AC, Gross KD, Clancy M, Lynch JA, Law LA, Lewis CE, Roemer FW, Powers CM, et al. The association between patella Alta and the prevalence and worsening of structural features of patellofemoral joint osteoarthritis: the multicenter osteoarthritis study. Arthritis Care Res (Hoboken). 2010;62:1258-65.

16. Kalichman L, Zhang Y, Niu J, Goggins J, Gale D, Felson DT, Hunter D. The association between patellar alignment and patellofemoral joint osteoarthritis features--an MRI study. Rheumatology (Oxford). 2007;46:1303-8.

17. Parikh SN, Lykissas MG, Gkiatas I. Predicting risk of recurrent patellar dislocation. Curr Rev Musculoskelet Med. 2018;11:253-60.

18. Elias JJ, Kilambi S, Goerke DR, Cosgarea AJ. Improving vastus medialis obliquus function reduces pressure applied to lateral patellofemoral cartilage. J Orthop Res. 2009;27:578-83.

19. Khan KM, Bonar F, Desmond PM, Cook JL, Young DA, Visentini PJ, Fehrmann MW, Kiss ZS, O'Brien PA, Harcourt PR, et al. Patellar tendinosis (jumper's knee): findings at histopathologic examination, US, and MR imaging. Victorian institute of sport tendon study group. Radiology. 1996; 200:821-7.

20. Reiff DB, Heenan SD, Heron CW. MRI appearances of the asymptomatic patellar tendon on gradient echo imaging. Skelet Radiol. 1995;24:123-6.

21. Cook JL, Khan KM, Kiss ZS, Purdam CR, Griffiths L. Prospective imaging study of asymptomatic patellar tendinopathy in elite junior basketball players. J Ultrasound Med. 2000;19:473-9.

22. Comin J, Cook JL, Malliaras P, McCormack M, Calleja M, Clarke A, Connell D. The prevalence and clinical significance of sonographic tendon abnormalities in asymptomatic ballet dancers: a 24-month longitudinal study. Br J Sports Med. 2013;47:89-92.

23. Draper CE, Besier TF, Fredericson M, Santos JM, Beaupre GS, Delp SL, Gold GE. Differences in patellofemoral kinematics between weight-bearing and non-weight-bearing conditions in patients with patellofemoral pain. J Orthop Res. 2011;29:312-7.

24. Magee DJ. Knee. In: Magee DJ, ed. Orthopedic physical assessment. 4th ed. Philadelphia, Pa: Saunders; 1997:661-763.

25. Laurin CA, Dussault $R$, Levesque HP. The tangential $x$-ray investigation of the patellofemoral joint: $x$-ray technique, diagnostic criteria and their interpretation. Clin Orthop Relat Res. 1979:16-26.

26. Wilson T. The measurement of patellar alignment in patellofemoral pain syndrome: are we confusing assumptions with evidence? J Orthop Sports Phys Ther. 2007;37:330-41.

27. Phillips CL, Silver DA, Schranz PJ, Mandalia V. The measurement of patellar height: a review of the methods of imaging. J Bone Joint Surg Br. 2010;92: 1045-53.

28. Grelsamer RP, Meadows S. The modified Insall-Salvati ratio for assessment of patellar height. Clin Orthop Relat Res. 1992:170-6.

\section{Publisher's Note}

Springer Nature remains neutral with regard to jurisdictional claims in published maps and institutional affiliations.

Ready to submit your research? Choose BMC and benefit from:

- fast, convenient online submission

- thorough peer review by experienced researchers in your field

- rapid publication on acceptance

- support for research data, including large and complex data types

- gold Open Access which fosters wider collaboration and increased citations

- maximum visibility for your research: over $100 \mathrm{M}$ website views per year

At BMC, research is always in progress.

Learn more biomedcentral.com/submissions 